\title{
Confusion in Giant Cell Arteritis - A Diagnostic Challenge
}

\author{
M. Maharaj, L. Shimon, D. Brake
}

Can J Neurol Sci. 2014; 41: 535-537

\section{SUMMARY}

A 70-year-old male with agitated confusion, ataxia, low grade fever and headache is presented. Inflammatory markers were elevated but extensive blood investigations, lumbar puncture and imaging studies failed to provide a diagnosis. Giant cell arteritis (GCA), also commonly known as temporal arteritis (TA) was suspected largely because of the persistently elevated inflammatory markers and awareness of atypical presentations in this disorder. The diagnosis was confirmed by temporal artery biopsy (TAB), which is considered the "Gold standard" in the investigation of this disorder.

Elevated erythrocyte sedimentation rate (ESR) and Creactive protein (CRP) in the elderly without other explanation, are highly sensitivity for GCA diagnosis. Presence of these markers even in atypical presentations in the elderly, require a high index of suspicion for the early diagnosis and management of GCA.

\section{Case Report}

A 70-year-old male was transferred from a community hospital for assessment by neurology for a progressive confusional state of one week. Two days prior to assessment, visual hallucinations and increased agitation were noted. One month before admission he developed headaches, photophobia, nausea, vomiting and gait unsteadiness. No visual symptoms were reported. Past history included an anxiety disorder, a right Adie's pupil, hypertension, chronic obstructive pulmonary disease (COPD), dyslipidemia, peripheral vascular disease with previous aorto-femoral bypass surgery and left calf fasciotomy for compartment syndrome. Medications included Diltiazem, Bisoprolol, Atorvastatin, Cyclobenzaprine, Roflumilast, Pantoprazole and tiotropium bromide puffers, all of which were held prior to the neurology assessment.

On physical exam the patient was afebrile with a blood pressure (BP) 110/60. He was agitated and physically aggressive with wandering, paranoid and confused behaviour. At one point he struck the examiner during the exam. Anisocoria was present with a poorly reactive right pupil at $4 \mathrm{~mm}$ and reactive left pupil at $2 \mathrm{~mm}$ diameter. Obvious focal signs, nuchal rigidity, and superficial temporal artery scalp tenderness were not present. The patient was unsteady in gait.

Midazolam $50 \mathrm{mg}$ IV and $10 \mathrm{mg}$ Haldol IM administered over 45 minutes failed to sedate the patient. Propofol boluses administered repeatedly intravenously to $160 \mathrm{mg}$ total dose, finally resulted in adequate sedation for lumbar puncture.
Other pertinent investigations included blood investigations which were remarkable for a microcytic anemia, ESR 73 $\mathrm{mm} /$ hour (Westergren), CRP $78 \mathrm{mg} / \mathrm{L}$, and LDH $1003 \mathrm{U} / \mathrm{L}$. Other blood investigations including WBC, platelets, D-Dimer, VDRL, ANA, ANCA, TSH, vitamin $\mathrm{B}_{12}$, calcium, thyroid antibodies, arterial blood gases, ammonia, LFT's, hepatitis, B and C serology and HIV serology were unremarkable. Computed tomogram (CT) head, abdomen and chest were normal.

Bone marrow exam, plus blood and urine cultures were negative. A trans thoracic echocardiogram was normal. An magnetic resnance imaging/angiography (MRI/MRA) (TOF)

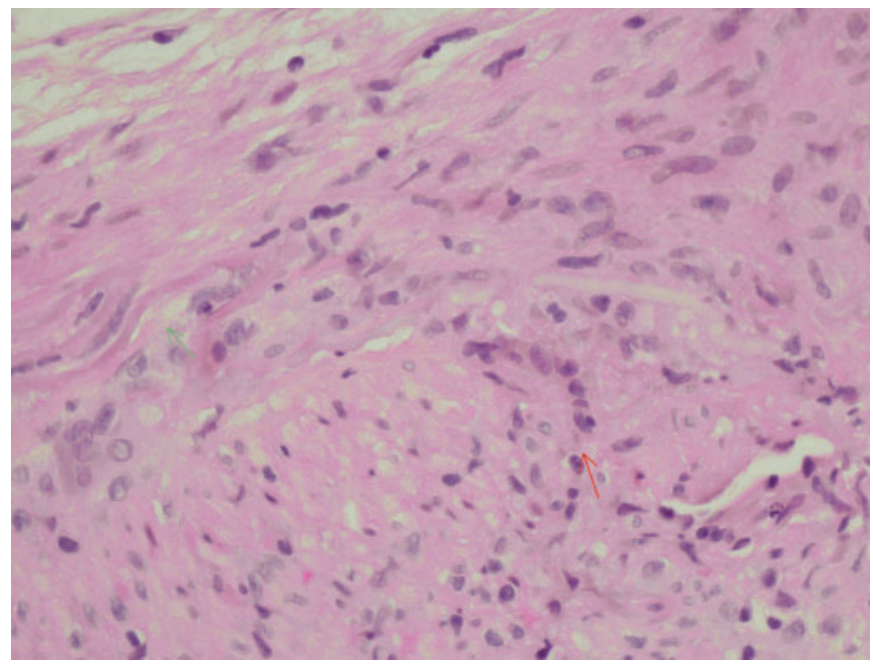

Figure: Higher magnification (20X) (H\& E stain) showing disrupted internal elastic lamina (green arrow) and multinucleated giant cell (red arrow).

From Internal Medicine/Neurology (MM, LS), Cape Breton Regional Hospital; Internal Medicine/Critical Care (DB), Health Park, Sydney, Nova Scotia, Canada. Received December 10, 2013. Final Revisions Submitted March 3, 2014. Correspondence to: M. Maharaj, Internal Medicine/Neurology, Cape Breton Regional Hospital, 1482 George Street, Sydney, Nova Scotia, B1P 1P3, Canada. Email: maharajm@cbdha.nshealth.ca. 
and magnetic resonance venogram (MRV) head showed post lumbar puncture (LP) dural enhancement and nonspecific white matter T2 hypertensities, but no evidence of stroke. No abnormalities of lumen calibre in the vertebrobasilar system or anterior circulation were noted. Imaging of extra cranial vessels was not performed. Cerebral spinal fluid (CSF) studies showed nucleated $\mathrm{WBC}$ of 2 , glucose of $2.9 \mathrm{mmol} / \mathrm{L}$ (serum glucose 5 $\mathrm{mmol} / \mathrm{L}$ ) and protein $0.71 \mathrm{gm} / \mathrm{L}$ (normal $0.6 \mathrm{gm} / \mathrm{L}$ ). Gram stain as well as acid fast bacillus (AFB) and fungal stains, polymerase chain reaction (PCR) for herpes simplex virus and CSF culture were negative. Mantoux test was negative.

Persistent agitation, confusion, and exacerbation of COPD resulted in intubation and admission to the intensive care unit (ICU). The patient was initially treated empirically with broad spectrum antibiotics but remained agitated, requiring sedation with a continuous Propofol infusion. Subsequently, neuroleptics, Midazolam and Morphine were used. Fever, despite antibiotics, ranging from 37.9 to 38.8 celsius was noted with ESR climbing to as high as $100 \mathrm{~mm} /$ hour and CRP $173 \mathrm{mg} / \mathrm{L}$, at the end of the first week of admission. Subsequently TAB in ICU whilst on ventilation was positive for TA (Figure). High dose intravenous methyl prednisolone over three days was started on tenth day of admission at the time of biopsy, followed by high dose oral steroids. Temperatures normalized in 24 hours and the patient was extubated within 48 hours. The delirium rapidly cleared over the ensuing week. The ESR and CRP normalized within three weeks of high dose steroids. Subsequent to discharge, an ophthalmology assessment found normal visual fields and unremarkable optic discs with no evidence of optic atrophy.

\section{Discussion}

Giant cell arteritis is a large and medium sized vessel vasculitis with a predilection for involvement of the cranial branches of the aortic arch. ${ }^{1}$ In $15 \%$ to $22 \%$ of patients, the aorta, subclavian, axillary and other branches may be involved., ${ }^{1,2}$ Survival is generally not affected but large vessel involvement with aortic aneurysm/dissection may increase morbidity and mortality. ${ }^{1,2}$ Permanent visual loss involving one or both eyes due to ischemic optic neuropathy is a feared complication in up to $20 \%$ of patients. ${ }^{1,2}$ It is mainly this potential for visual loss that makes early diagnosis imperative. Steroid treatment is usually highly effective in its prevention, but not reversal. Infrequently, further visual deterioration after beginning therapy has been reported..$^{1,2}$

Typical symptoms of GCA include headache in two thirds of patients, visual impairment or blindness, jaw claudication and superficial temporal artery tenderness with a female predominance. . $^{1,3}$

Constitutional symptoms including fever, sweat, malaise, anorexia and weight loss reportedly occurs in half of presentations. Polymyalgia rheumatica may occur in approximately $40 \%$ of cases. ${ }^{1}$ Neurological manifestations such as stroke and cranial neuropathies occur in approximately a third of the cases. ${ }^{1}$

Confusion as a dominant symptom at presentation has been reported with variable postulated causes including stroke from vertebrobasilar ischemia and vertebrobasilar arteritis. ${ }^{4}$ Ischemic stroke occurs in $3-4 \%$ of $\mathrm{GCA}^{5}$. Luminal irregularities, occlusions and filling defects suggestive of thrombus have been documented on imaging in a previous study. There is a predilection for vertebral arteries. ${ }^{4,5}$ These abnormalities of a direct brain insult were not identified and therefore cannot explain this striking presentation of agitated delirium. In GCA, peripheral inflammation is responsible for inflammatory cytokines production, including interferon gamma, interleukin 1, interleukin 6 and TNF alpha. Different concentration of these cytokines may correlate with different clinical presentations in GCA.$^{1,2}$ Inflammatory cytokines have been implicated as part of an aberrant stress response in the pathophysiology of delirium. ${ }^{6}$ We speculate that the effect of the inflammatory cytokines on a vulnerable aging brain is responsible for the prominent delirium, in the absence of another explanation. ${ }^{6}$

Although headache was an initial presenting symptom in our case, in as much as one third of patients atypical presentations of neurological manifestations may occur, ${ }^{1}$ which may lead to delayed diagnoses. ${ }^{1}$ Published criteria in the American College of Rheumatology $(\mathrm{ACR})^{3}$ is unhelpful in individual cases and may miss approximately $25 \%$ of biopsy proven cases with a false positive rate of up to $28 \% .^{7}$ The relevance of inflammatory markers in consideration of this diagnosis cannot be overstated but like the ARC criteria, that too can have its pitfalls. Up to $23.5 \%$ of patients with GCA may have a normal ESR. ${ }^{1,8} \mathrm{C}$ reactive protein negative disease with elevated ESR occurs, but is much lower at $1.7 \%$. Double false negative inflammatory markers are rare at $0.8 \%$ but both elevated ESR and CRP are highly sensitivity at $99 \% .^{8}$

It was the persistence of both elevated inflammatory markers without other explanation that lead to the diagnostic TAB. This test remains the "gold standard" in the appropriate clinical setting, with a sensitivity up to $90 \% 1,7,9$ Treatment with corticosteroids in a timely fashion is highly effective in this condition..$^{1,2}$ In our case, steroids were started just before TAB but nonetheless delayed. It is worth pointing out that clinicians generally accept TAB should be performed within two weeks of starting steroid treatment. ${ }^{9}$ There is data from a small case series, that $82 \%$ of patients will have a positive TAB, even if performed between four to six weeks. ${ }^{9}$

Giant cell arteritis may not be an evident diagnosis. Although our patient met the ACR criteria at presentation to neurology, the atypical features of a dominantly confusional state, ataxia and pyrexia of unknown origin presented a complex clinical picture. Management difficulties encountered with the patient's agitated delirium and the extensive work up required to exclude multiple potential differential diagnoses, contributed to a delay in diagnosis. Elevated and unexplained inflammatory markers in typical or atypical presentations, have a high sensitively for GCA and should warrant a high index of suspicion for early diagnosis and management.

\section{ACKNOWLEDGEMENTS}

The authors thank Doctor S. Mansoor for the pathology image and Professor C.E. Maxner for comments on the manuscript.

Dr. Maharaj and Dr. Brake are Assistant Professors and Dr. Shimon is a Lecturer at Dalhousie University. 


\section{REFERENCES}

1. Salvarani C, Cantini F, Boiardi L, Hunder GG. Polymyalgia rheumatica and giant-cell arteritis. N Engl J Med. 2002;347(4): 261-70

2. Cid MC, Garcia-Martinez A, Lozano E, Espigol-Frigole G, Hernandez-Rodriguez J. Five clinical conundrums in the management of giant cell arteritis. Rheum Dis Clin North Am. 2007;33(4):819-34.

3. Hunder GG, Bloch DA, Michel BA, et al. The American college of rheumatology 1990 criteria for the classification of giant cell arteritis. Arthritis Rheum. 1990;Aug;22(8):1122-8.

4. Zeidler M, Hughes T, Zeman A. Confused by arteritis. Lancet. 2000;355:374-5.

5. Tang V, Fantaneanu T, Charkaborty S, Patel V, Dowlatshahi D. Intracranial non-occlusive thrombus and multiple strokes in giant cell arteritis. Can J Neurol Sci. 2012;(1):116-17.
6. Maculluch AMJ, Anand A, Davis DHJ, et al. New horizons in the pathogenesis assessment and management of delirium. Age Ageing. 2013;42:667-74.

7. Murchison AP, Gilbert ME, Bilyk JR, et al. Validity of the American college of rheumatology criteria for the diagnosis of giant cell arteritis. Am J Ophthalmol. 2012;154:722-9.

8. Parikh M, Miller NR, Lee AG, et al. Prevalence of a normal creactive protein with an elevated erythrocyte sedimentation rate in biopsy-proven giant cell arteritis. Amer Academy Ophthalmol. 2006;113:1842-5.

9. Ray-Chaudhuri N, Ah Kine D, Tijani SO, et al. Effect of prior steroid treatment on temporal artery biopsy findings in giant cell arteritis. J Ophthalmol. 2002;86:530-2. 